\title{
不变结构半弹道式再入飞行器的混合状态估计 与模型集设计
}

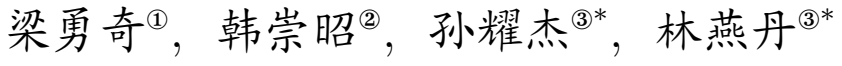 \\ (1) 西安交通大学航天学院, 西安 710049 \\ (2) 西安交通大学智能网络与网络安全教育部重点实验室, 机械制造系统工程国家重点实验室, 电信学院综合自动化研究所, \\ 西安 710049 \\ (3) 复旦大学光源与照明工程系, 先进照明技术教育部工程研究中心, 上海 200433 \\ * 通信作者. E-mail: yjsun@fudan.edu.cn, ydlin@fudan.edu.cn
}

收稿日期: 2009-09-13; 接受日期: 2010-05-11

国家重点基础研究发展计划 (批准号: 2007CB311006, 2010CB734102)、国家自然科学基金创新研究群体科学基金 (批准号: 60921003) 和科技部科技特派员项目 (批准号: 2009GJC00008) 资助

\begin{abstract}
摘要 多模型方法是混合估计的主流方法之一, 以该方法估计不变结构半弹道式再入飞行器 (SBRV) 混合状态的难点在于设计有效的模型集. 文中提出了一种伪 Monte Carlo 模型集可以使 SBRV 混合 状态估计器在最小均方差 (MMSE) 意义下接近于最优. SBRV 的再入估计具有高度的非线性, 同时其 模式由多个边界已知的参数张成. 给出了这种伪 Monte Carlo 模型集的设计方法, 并分析了其性能特 征. 该模型集相比于 Monte Carlo 方法生成的模型集有更高的精度, 理论分析和仿真结果表明了新设 计模型集的有效性与合理性.
\end{abstract}

关键词半弹道式再入飞行器 混合估计 模型集设计 Monte Carlo 法 伪 Monte Carlo 法

\section{1 引言}

基于雷达量测对再入体实时跟踪是目标跟踪领域的重要问题, 传统研究的重点是弹道式再入飞行 器 $(B R V)^{[1-4]}$, 该飞行器结构对称, 同时其轨迹由再入的初始状态决定. 然而再入体的结构通常不对 称, 该不对称产生的升力导致飞行器轨迹的复杂以及控制、估计及预测的困难 ${ }^{[5-10]}$, 这种类型的飞行 器称作半弹道式再入飞行器 (SBRV, semi-ballistic reentry vehicle), 例如航天飞机、宇宙飞船、碎片、 飞行器残骸、一些卫星和一些类别的导弹. SBRV 即能以不变结构的方式再入, 也能以可变结构的方 式再入, 对前者的状态估计是后者的基础, 在不考虑飞行器旋转的情况下本文研究不变结构 SBRV 的 估计问题.

SBRV 的状态由连续成分和离散成分组成, 连续成分称作基态, 指的是一般系统的状态, 离散成分 称作模式, 因而 SBRV 的估计在本质上属于混合估计, 混合估计的一个主流方法为多模型方法 ${ }^{[11,12]}$. 该方法需要设计一组模型以覆盖系统可能的模式与行为, 然后通过这些模型输出的组合得到多模型 方法的输出. 多模型方法的性能很大程度上依赖于模型集, 模型集的设计又是多模型方法应用最主要 
的问题 ${ }^{[13]}$, 所以本文着重探讨 SBRV 多模型估计器的模型集设计.

模型集的设计不但重要而且很难, 当前的设计大多缺乏足够理论依据或依赖于极高技巧 ${ }^{[13]}$, 模 型集拥有固定的结构或者可变的结构. 前者的应用最为广泛, 这种模型集用于自治多模型 (AMM, autonomous multiple-model) 方法或协作多模型 (CMM, cooperative multiple-model) 方法 ${ }^{[11,14,15]}$. AMM 估计器的每个模型各自独立地工作, 该估计器相比于非多模型估计器的优点在于其输出是对各模型输 出结果的优化处理 ${ }^{[11]}$. 本文使用 AMM 法估计不变结构 SBRV 的混合状态.

Magill 使用了与模式空间相等的模型集, 从而得到最小均方差 (MMSE, minimum mean square error) 意义下的最优估计器 ${ }^{[14]}$; 实际上模型集通常要比模式空间小很多 ${ }^{[16,17]}$, 针对这种情况的通用 固定结构模型集设计方法很少, 在确知各个参数有界取值区间的条件下, 文献 [18] 给出了一种系统的 模型集设计方法, 该方法只针对线性系统. 文献 [4] 基于参数的边界信息设计模型集以估计非线性系统 的状态, 但是只考虑了一维模式空间情形. $\mathrm{Li}$ 对模型集设计提供了一个理论描述: 真实模式和模型被 描述都为概率分布的先验信息已知的随机变量, 从而模型集的设计成为设计模型以近似模式的分布. 在此基础上, Li 提出了 3 种模型集设计途径 ${ }^{[18]}$. 作为对文献 [18] 的进一步发展, 文献 [19] 在多维的 模式空间中给出了与模式分布失配最小的模型集.

虽然多模型方法广泛地用于混合估计, 该方法在再入问题上的应用很少, 其难度在于模型集的设 计，而且上述模型集设计方法都不符合 SBRV 的特征. SBRV 具有高度非线性，则无法使用文献 [17] 中的模型集设计方法; 其模式空间由多个边界已知的参数张成, 又无法使用文献 [4] 的给出的设计; 这 些参数仅知其散布的区间, 而其分布通常未知, 所以也无法使用文献 $[18,19]$ 中给出的模型集设计方法. 本文设计的模型集符合 SBRV 的上述要求, 这些模型散布在 SBRV 的模式空间, 其使用方便, 而且可 以使估计器在 MMSE 意义下接近于最优.

本文内容组织如下: 第 2 节介绍 SBRV 模型 (关于模型设计较为详细的内容已在文献 [9] 中给出), 运动特征及模式特征; 第 3 节设计 SBRV 最小均方差 (MMSE) 准则下接近于最优的混合状态估计器; 第 4 节提出伪 Monte Carlo 模型集并分析该模型集的理论精度, 实现方法以及性能特征; 第 5 节是仿 真结果; 结论在第 6 节给出.

\section{$2 \mathrm{SBRV}$ 的建模及运动和模式分析}

SBRV 具有不对称的外形或结构, 其外形的不对称可视作结构的不对称. 在再入的过程中该飞行 器主要承受重力与气动力, 重力作用于质心 $O, O^{\prime}$ 是气动力作用的中心, 如图 1 所示. 气动力分解为 升力和阻力. 当质心 $O$ 在轴线 $A B$ 上时升力为零, 当 $O$ 与 $A B$ 不重合时升力存在. 升力来源于 SBRV 结构的不对称性, 并根据物理意义分解为爬升力与转弯力, 前者控制飞行器的爬升与俯冲, 后者控制飞 行器的左转与右转; 爬升力垂直于阻力和转弯力, 转弯力平行于当地水平面并垂直于阻力. 阻力与速 度的方向相反, 当升力存在时, 阻力中有诱导阻力生成. 由单位转弯力向量、单位爬升力向量和单位速 度向量组成的正交坐标系称为半速度坐标系 (文献 [1] 中称为 VTC 框架). 上述力的关系如图 1 所示.

将 SBRV 看作质点 (如图 1 所示, 该质点与质心重合), 其状态在东北天 (ENU, earth north up) 坐 标系中描述, 将气动力从半速度坐标系转换到 ENU 坐标系, 并考虑离心力与 Coriolis 力的影响, 可得 到 SBRV 模型

$$
X_{(k+1)}=F X_{k}+G f\left(k, X_{k}\right)+w_{k},
$$




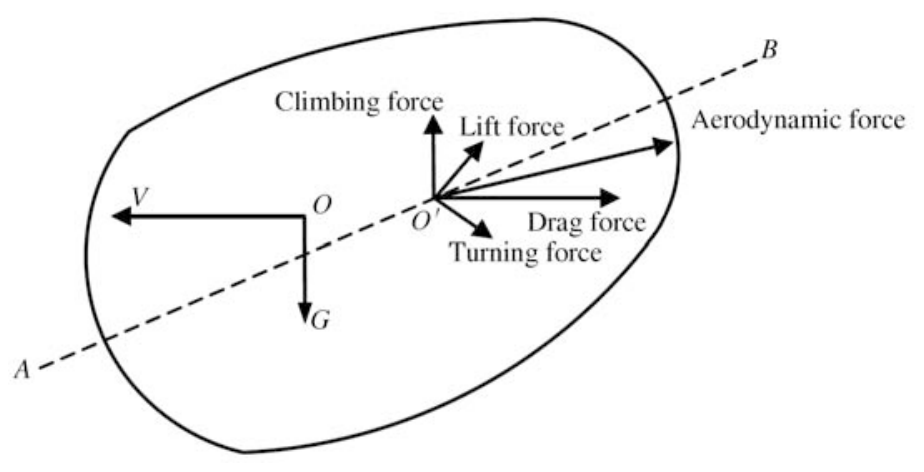

图 1 半弹道式再入飞行器及气动力分析

Figure 1 SBRV and geometry of forces

其中

$$
\begin{gathered}
X_{(k+1)}=\left[x_{(k+1)} \dot{x}_{(k+1)} y_{(k+1)} \dot{y}_{(k+1)} z_{(k+1)} \dot{z}_{(k+1)}\right]^{\prime}, \quad w_{(k)}=\left[w_{x} \dot{w}_{v x} w_{y} w_{v y} w_{z} w_{v z}\right]^{\prime}, \\
F=\left[\begin{array}{cccccc}
1 & T & 0 & 0 & 0 & 0 \\
0 & 1 & 0 & 0 & 0 & 0 \\
0 & 0 & 1 & T & 0 & 0 \\
0 & 0 & 0 & 1 & 0 & 0 \\
0 & 0 & 0 & 0 & 1 & T \\
0 & 0 & 0 & 0 & 0 & 1
\end{array}\right], \quad G=\left[\begin{array}{ccc}
\frac{T^{2}}{2} & 0 & 0 \\
T & 0 & 0 \\
0 & \frac{T^{2}}{2} & 0 \\
0 & T & 0 \\
0 & 0 & \frac{T^{2}}{2} \\
0 & 0 & T
\end{array}\right],
\end{gathered}
$$

$$
\begin{aligned}
& f\left(k, X_{k}\right)= \\
& {\left[\begin{array}{c}
-\frac{1}{2} \alpha_{d} \rho \sqrt{\dot{x}_{k}^{2}+\dot{y}_{k}^{2}+\dot{z}_{k}^{2}} \dot{x}_{k}-\frac{1}{2} \alpha k_{t d} \rho\left(\dot{x}_{k}^{2}+\dot{y}_{k}^{2}+\dot{z}_{k}^{2}\right) \frac{\dot{y}_{k}}{\sqrt{\dot{x}_{k}^{2}+\dot{y}_{k}^{2}}}-\frac{1}{2} \alpha k_{c d} \rho \sqrt{\dot{x}_{k}^{2}+\dot{y}_{k}^{2}+\dot{z}_{k}^{2}} \frac{\dot{x}_{k} \dot{z}_{k}}{\sqrt{\dot{x}_{k}^{2}+\dot{y}_{k}^{2}}} \\
-\frac{1}{2} \alpha_{d} \rho \sqrt{\dot{x}_{k}^{2}+\dot{y}_{k}^{2}+\dot{z}_{k}^{2}} \dot{y}_{k}+\frac{1}{2} \alpha k_{t d} \rho\left(\dot{x}_{k}^{2}+\dot{y}_{k}^{2}+\dot{z}_{k}^{2}\right) \frac{\dot{x}_{k}}{\sqrt{\dot{x}_{k}^{2}+\dot{y}_{k}^{2}}}-\frac{1}{2} \alpha k_{c d} \rho \sqrt{\dot{x}_{k}^{2}+\dot{y}_{k}^{2}+\dot{z}_{k}^{2}} \frac{\dot{y}_{k} \dot{z}_{k}}{\sqrt{\dot{x}_{k}^{2}+\dot{y}_{k}^{2}}} \\
-\frac{1}{2} \alpha_{d} \rho \sqrt{\dot{x}_{k}^{2}+\dot{y}_{k}^{2}+\dot{z}_{k}^{2}} \dot{z}_{k}+\frac{1}{2} \alpha k_{c d} \rho \sqrt{\dot{x}_{k}^{2}+\dot{y}_{k}^{2}+\dot{z}_{k}^{2} \sqrt{\dot{x}_{k}^{2}+\dot{y}_{k}^{2}}}
\end{array}\right]} \\
& -\frac{\mu}{x_{k}} \\
& -\omega_{k}\left[\begin{array}{c}
\left.y_{k}^{2}+\dot{y}_{k}^{2}+\left(\dot{z}_{k}+a\right)^{2}\right)^{3 / 2} \\
z_{k}+a
\end{array}\right] \\
& {\left[\begin{array}{c}
-\sin \left(\omega_{k}\right)^{2} y_{k}+\cos \left(\omega_{k}\right) \sin \left(\omega_{k}\right)\left(z_{k}+a\right) \\
\cos \left(\omega_{k}\right) \sin \left(\omega_{k}\right) y_{k}-\cos \left(\omega_{k}\right)^{2}\left(z_{k}+a\right)
\end{array}\right]-2 \omega\left[\begin{array}{c}
-\sin \left(\phi_{k}\right) \dot{y}_{k}+\cos \left(\phi_{k}\right) \dot{z}_{k} \\
\sin \left(\phi_{k}\right) \dot{x}_{k} \\
-\cos \left(\phi_{k}\right) \dot{x}_{k}
\end{array}\right],}
\end{aligned}
$$

$k_{c d}$ 是爬升力与阻力的比值, $k_{t d}$ 是转弯力与阻力的比值, $\mu$ 是地球引力常数, $\omega$ 是地球角速度的大小, $\phi_{k}$ 是飞行器的纬度, $a$ 是 ENU 坐标系的原点到地心的距离, 其值为地球平均半径加 ENU 坐标系原点的 海拔高度, 则飞行器到地心的距离为 $\left(x_{k}^{2}+y_{k}^{2}+\left(z_{k}+a\right)^{2}\right)^{1 / 2}, \rho$ 是大气密度, 阻力参数 $\alpha_{d}=\left(1+c k^{2}\right) \alpha$, 升阻比 $k$ 为升力与阻力的比值, $k^{2}=k_{t d}^{2}+k_{c d}^{2}, c$ 是与诱导阻力相关的系数, $\alpha=\left(S C_{D}\right) / m$ 由飞行器质 


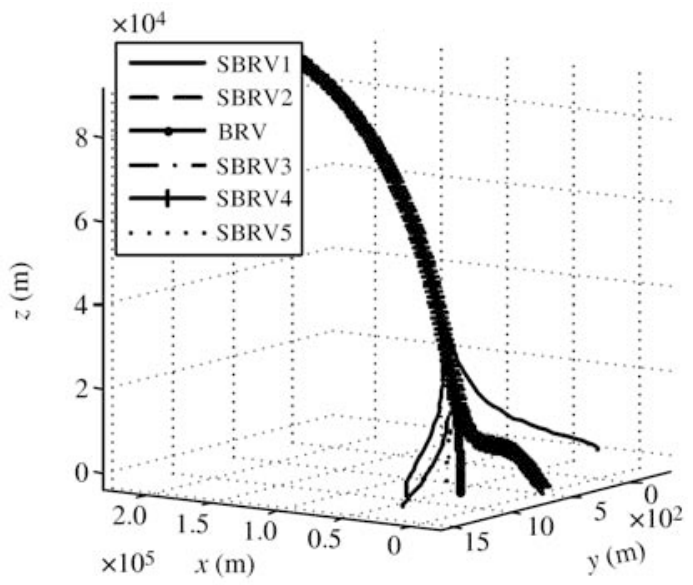

图 2 不变结构 $\mathrm{SBRV}$ 的三维再入轨迹

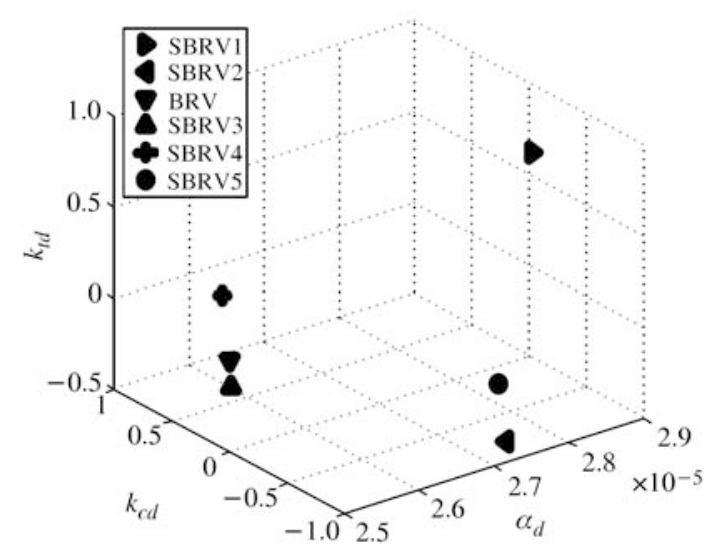

图 3 各轨迹对应的模式

Figure 2 Three-dimensional trajectories of a invariable-structure SBRV

Figure 3 The corresponding mode of each trajectory

量 $m$, 参考面积 $S$ 和阻力系数 $C_{D}$ 决定. $C_{D}$ 依赖于速度、飞行器距离地面的高度、飞行器的长直比 和空气的粘性系数等 ${ }^{[5]}$. 当 $k_{t d}=0$ 且 $k_{c d}=0$ 时, 该模型就是 BRV 模型.

不变结构 SBRV 的轨迹受到升阻比的影响, 该值主要取决于 SBRV 的结构, 其范围可先验获知. 航天飞机升阻比可以大于 1 , 旋成体升阻比小于 0.8 , 宇宙飞船的升阻比不超过 0.5 , 而细长体的升阻比 小很多. 在给定的初始状态下, BRV 的轨迹是确定的, BRV 阻力系数的值通常假设为定常 ${ }^{[3,4]}$; 根据 这一假设, SBRV 的轨迹受到 $k_{c d}$ 与 $k_{t d}$ 影响, 其阻力受到诱导阻力的影响. 这些参数代表了 SBRV 的 特征, 它们的值代表了该飞行器的模式, 所有可能模式的集合构成了模式空间. 所以 SBRV 轨迹的不 确定性主要由 $k_{t d}, k_{c d}$, 和 $\alpha_{d}$ 张成的空间决定, 这一空间就是 SBRV 的模式空间.

图 2 是 SBRV 的一些轨迹, 图 3 是这些轨迹对应的模式, 相关参数是: $x_{0}=232000 \mathrm{~m}, y_{0}=0 \mathrm{~m}$, $z_{0}=88000 \mathrm{~m}, \gamma_{0}=190^{\circ}, v_{0}=2290 \mathrm{~m} / \mathrm{s}, \alpha_{d}=\frac{9.8}{40000} \mathrm{~m}^{2} / \mathrm{Kg}, k_{c d} \in(-0.8,0.8), k_{t d} \in(-0.8,0.8)$. 不考虑 过程噪声. 每一条轨迹对应模式空间中的一个点, 同一轨迹在图 2 与 3 中以相同的名称表示. 从图中 可见, BRV 轨迹是 SBRV 轨迹的特例, 其模式是 SBRV 模式空间中一个确定的点. 给定了初始状态, $\mathrm{BRV}$ 轨迹的长度、最大过载以及落点都是确定的; 在阻力系数不变的假设下, 不变结构 SBRV 的模式 在整个再入过程中不变, 通过初始结构的选择, SBRV 可以 “设计” 轨迹的长度、最大过载和落点.

\section{3 估计器的设计}

混合状态估计器由基态估计器和模式估计器组成. 为了设计最优的基态估计器, 一个基本的想法 是以均方差作 (MSE) 为估计器的性能指标, 在 Bayesian 估计下使该指标最小化则得到最小均方差 (MMSE) 估计器

$$
\hat{X}^{\mathrm{MMSE}}=\arg _{\hat{X}}^{\min } E\left\{(X-\hat{X})^{\prime}(X-\hat{X}) \mid Z^{k}\right\}=\int_{\Omega} X f\left(X \mid Z^{k}\right) \mathrm{d} X,
$$

其中 $\Omega$ 是状态空间, $Z^{k}=\left\{Z_{1}, \ldots, Z_{k}\right\}$ 表示从初始时刻到当前时刻所有量测的集合, $f\left(X \mid Z^{k}\right)$ 是给定量 测下基态向量 $X$ 的后验概率密度函数. 考虑到 SBRV 状态估计的复杂性来源于模式的未知, (2) 式可 
以表示为

$$
\begin{aligned}
\hat{X}^{\mathrm{MMSE}} & =\int_{\Omega} X \int_{\alpha} \int_{k_{c d}} \int_{k_{t d}} f\left(X, \alpha, k_{c d}, k_{t d} \mid Z^{k}\right) \mathrm{d} k_{t d} \mathrm{~d} k_{c d} \mathrm{~d} \alpha \mathrm{d} X=\int_{\Omega} X \int_{\mathrm{S}} f\left(X, \Theta \mid Z^{k}\right) \mathrm{d} \Theta \mathrm{d} X \\
& =\int_{\mathrm{S}} f\left(\Theta \mid Z^{k}\right) \int_{\Omega} X f\left(X \mid \Theta, Z^{k}\right) \mathrm{d} X \mathrm{~d} \Theta=\int_{\mathrm{S}} \hat{X}(\Theta) f\left(\Theta \mid Z^{k}\right) \mathrm{d} \Theta,
\end{aligned}
$$

其中 $\Theta=\left\{\alpha, k_{c d}, k_{t d}\right\}$, 积分域为模式空间 S. 通过数值积分近似 (3) 式

$$
\hat{X}^{\mathrm{MMSE}} \approx \sum_{i} \hat{X}\left(\Theta_{i}\right) p\left(\Theta_{i} \mid Z^{k}\right),
$$

其中 $\mathrm{S}$ 为在整个再入过程中保持不变的 $M$ 维模式空间; 将其划分为一系列子空间 $\mathrm{S}=\bigcup_{i=1}^{R} \mathrm{~S}_{i}, \forall i \neq j$ 且 $i, j \in\{1, \ldots, R\}$, 则 $\mathrm{S}_{i} \cap \mathrm{S}_{j}=\phi, \Theta_{i}$ 在 $\mathrm{S}_{i}$ 的中心, 模型 $m_{i}$ 根据 $\Theta_{i}$ 的值构造得到, $\hat{X}\left(\Theta_{i}\right)$ 是模型 $m_{i}$ 的估计结果, $p\left(\Theta_{i} \mid Z^{k}\right)$ 表示该模型的后验概率. (4) 式中的模型集为 $\mathrm{M}=\left\{m_{1}, \ldots, m_{R}\right\}$.

与 (2) 式类似, 未知模式由下式估计

$$
\hat{s}^{\mathrm{MMSE}}=\int_{\mathrm{S}} \Theta f\left(\Theta \mid Z^{k}\right) \mathrm{d} \Theta .
$$

通过数值计算近似 (5) 式的值

$$
\hat{s}^{\mathrm{MMSE}} \approx \sum_{i} \Theta_{i} p\left(\Theta_{i} \mid Z^{k}\right) .
$$

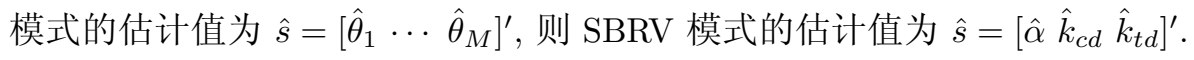

文献 [14] 讨论了 (3) 和 (5) 式的一种特殊情况, 当模式空间为一维而且只包含有限个模式时文献 [14] 使用了与模式空间 $S$ 相等的模型集 $M, M=S$. 对于一般情形 $(S>>M)$, 在模式分布的先验信息已 知时根据文献 $[18,19]$ 提供的方法设计模型集; 当仅知模式分布的区间信息时, 文献 [17] 给出一种线 性系统的模型集设计方法. 因而 SBRV 所代表的非线性系统需要寻找新的模型集设计方法. (4) 和 (6) 式表明多模型方法的估计结果接近于 MMSE 估计器 (3) 和 (5) 式, 其精度主要地取决于设计于模式 空间的模型集 ${ }^{[13]}$.

(4) 和 (6) 式组成了 SBRV 的混合状态估计器, 它们反映出多模型估计器的精度除了受到模型集 的影响, 实际还受到每个成员滤波器以及在相应模型为真实模型的假设下的后验概率的影响. 可用于 再入问题的滤波器已得到较为系统的研究 ${ }^{[1-4]}$, 而后验概率的值通过 Bayesian 公式近似估计 ${ }^{[11,14]}$, 所以我们的重点是如何设计模型集.

\section{4 伪 Monte Carlo 模型集}

在 MMSE 意义下, 文献 [14] 给出了最优估计, 通过 (4) 和 (6) 式对 (3) 和 (5) 式的离散表明该估 计器对 (3) 和 (5) 式的近似主要取决于模型集的设计, 即就是对模式空间的离散化. SBRV 的模式空 间由阻力参数 $\alpha$ 与两个比值 $k_{t d}$ 和 $k_{c d}$ 确定, 假设各个参数相互独立, 则 SBRV 需要在三维立方体中 进行模型集的设计.

Monte Carlo 法为数值积分的常用方法, 通过该方法均匀地从积分区域中抽样得到离散的点, 基于 这些点生成的模型集称作 Monte Carlo 模型集. Monte Carlo 方法均匀地从模式空间生成 $R$ 个模型, 该方法在理论上使 (4) 与 (6) 式的误差可达到 $O\left(R^{-1 / 2}\right)$. Monte Carlo 模型集的缺点主要是这些模型 随机产生, 因而其误差 $O\left(R^{-1 / 2}\right)$ 不是确定的, 而是概率意义的. 为了克服这一矛盾, 本文引进基于数 论方法的模型集, 其误差相比于 Monte Carlo 模型集不但更低而且是绝对误差. 


\section{1 模型集均匀度的度量}

引理 1 对于包含 $M$ 个参数的模型集 $\mathrm{M}$, 估计器 (4) 和 (6) 式的误差不会高于 $C 2^{M} D^{\star}(\mathrm{M})^{[20]}$, 其中 $D^{\star}(\mathrm{M})$ 是模型集的星偏差, $C$ 分别由基态估计器和模式估计器决定. 对于势为 $R$ 的模型集, (4) 和 (6) 式的误差不会低于 $O\left(R^{-1}\right)^{[21]}$. 对于数论方法生成的模型集, (4) 和 (6) 式的误差不会低于 $C(M) \frac{(\log R)^{(M-1) / 2}}{R}[22]$.

对多重数值积分, 引理 1 已经在文献 [20-22] 中已得到证明. 在不考虑各个模型使用的滤波器以及 Bayesian 后验概率近似的条件下, 引理 1 对估计器 (4) 和 (6) 式成立. 该引理给出混合估计器精度的上 界与下界, 同时表明星偏差是衡量点集散布的有效指标, 星偏差的值越小, 积分的误差越小. 基于引理 1 , 王元指出 $\forall \epsilon>0$, 基于数论方法生成的点集可以使数值积分 (4) 和 (6) 式的误差达到 $O\left(R^{-1+\epsilon}\right)^{[23]}$. 因而数论方法生成的模型集可以使估计器的性能接近于最优. 正因为此, 本文对估计器 (3) 和 (5) 式 使用数论方法生成的确定性模型集, 其精度高于相等势的 Monte Carlo 模型集.

Korobov, 华罗庚, 王元, Hlawka, Halton 和 Niederreiter 等做了许多工作证明了这种均匀分布点集 的可能性与存在性. 华罗庚与王元研究了如何在多维子空间中生成这种均匀散布的点, 并给出了一系

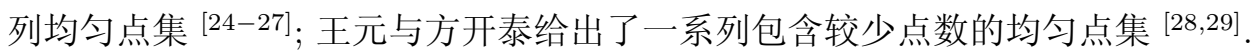

在模式空间中基于均匀度准则构造的取代 Monte Carlo 模型集的确定的模型集称作伪 Monte Carlo 模型集. 对于本文来说, 模型集均匀度由数论中最常用到的星偏差衡量 ${ }^{[27,30]}$. 模型集的星偏差表示为

$$
D^{\star}(\mathrm{M})=\operatorname{Sup}_{\Theta \in \mathrm{S}}\left|\frac{N(\mathrm{M}, \Theta)}{R}-\frac{\operatorname{Vol}(0, \Theta)}{\operatorname{Vol}(\mathrm{S})}\right|,
$$

其中 $\mathrm{M}=\left\{m_{1}, \cdots, m_{R}\right\}, R$ 表示模型集的势, $M$ 表示 $\Theta$ 中包含参数的数量, 各参数相互独立, $[0, \Theta)=$ $\left[0, \theta_{1}\right) \times \cdots \times\left[0, \theta_{M}\right)$ 表示原点与向量 $\Theta$ 确定的矩形, $\operatorname{Vol}(0, \Theta)$ 表示其体积, $N(\mathrm{M}, \Theta)$ 表示该矩形包含 的模型数量, $\operatorname{Vol}(\mathrm{S})$ 表示模式空间的体积. 星偏差反映了模型集的不均匀度, 该值越小表明模型集均匀 度越高.

该模型集对应参数值构成的矩阵称作模型集矩阵, 模型集矩阵的元素由各参数离散水平的序号 表示; 该矩阵每一行表示一个模型所对应参数的值, 每一列表示相应参数离散水平序号的排列. 如 果模型集矩阵的 $M_{1}$ 个参数每个有 $N_{1}$ 个水平, $M_{2}$ 个参数每个有 $N_{2}$ 个水平, 则该模型集表示为 $\mathrm{M}\left(R ; N_{1}^{M_{1}}, N_{2}^{M_{2}}\right)$, 以此类推.

\section{2 伪 Monte Carlo 模型集的构造}

基于数论方法设计伪 Monte Carlo 模型集, 首先需要将各个参数离散化, 然后在所有参数离散水 平的基础上构造均匀散布在模式空间中的模型集. 若在 $M$ 维模式空间中设计 $R$ 个模型, 任意参数 $\theta_{i} \in \Theta$ 的离散水平由下式得到

$$
\theta_{i j}=\theta_{i}^{-}+\frac{2 j-1}{2 N_{i}}\left(\theta_{i}^{+}-\theta_{i}^{-}\right), j=1, \ldots, N_{i},
$$

其中 $N_{i}$ 是 $\theta_{i}$ 的离散水平数, $r_{i}=R / N_{i}$ 表示 $\theta_{i}$ 各离散水平在模型集中出现的次数, $\bmod \left(R, N_{i}\right)=0$, $i \in\{1, \ldots, M\}, \theta_{i}^{+}$和 $\theta_{i}^{-}$是 $\theta_{i}$ 所在区间的上界和下届. 如果所有离散水平的组合都加以考虑, 该模型 集中有 $N_{1} N_{2} \cdots N_{M}$ 个模型, 这个数量无疑太大, 需要从中选择出数量较少并且均匀散布的模型集.

根据数论方法生成伪 Monte Carlo 模型集的方法主要有两种, 第一种是 Korobov ${ }^{[31]}$ 与 Hlawka ${ }^{[32]}$ 提出, 由华罗庚与王元 $[27]$ 等发展, 其生成方式为

$$
P_{N}(\mathrm{M})=\left(k a_{1}, k a_{2}, \ldots, k a_{E(N)}\right)(\bmod N), k=1, \ldots, N,
$$

316 
其中 $P_{N}(\mathrm{M})$ 表示模型集矩阵, 各参数的离散水平数相等: $N_{1}=\cdots=N_{M}=N$, 模型集的势 $R=N$, $\left(a_{1}, \ldots, a_{M}\right)$ 的选择基于模型集的均匀度, $a_{1}=1, a_{i}$ 为整数, $\left(a_{i}, N\right)=1$ 并且 $1<a_{i}<N(2 \leqslant i \leqslant N)$, $a_{i} \neq a_{j}(i \neq j) . E(N)$ 表示 $(11)$ 式生成模型集包含参数的最大数量

$$
E(N)=N \prod_{i=1}^{s}\left(1-\frac{1}{p_{i}}\right),
$$

其中 $p_{1}<p_{2}<\cdots<p_{s}$ 是不同的素数, $E(N) \leqslant N-1$, 当 $N$ 为素数时等号成立. 如果模型集矩阵包 含的参数个数 $M \leqslant E(N)$, 则需要从 $P_{N}(\mathrm{M})$ 中选取均匀度最高的列组成模型集, 基于均匀度准则从 $E(N)$ 中选取 $M$ 个列共有 $C_{E(N)}^{M}$ 种可能性, 其计算量通常太大.

当 $N$ 是素数时采用第二种模型集生成方法, 这种方法是 Korobov 提出 ${ }^{[33]}$, 由华罗庚和王元 ${ }^{[27]}$, 王元和方开泰 ${ }^{[28]}$, Niederreiter ${ }^{[30]}$ 等大力发展.

$$
P_{N}(\mathrm{M})=\left(k b^{0}, k b^{1}, \ldots, k b^{M-1}\right)(\bmod N), k=1, \ldots, N,
$$

其中 $N_{1}=\cdots=N_{M}=N, E(N)=N-1, b$ 是整数且 $1<b<N, b^{i} \neq b^{j}(\bmod N)(i \neq j), b$ 的值通 常为原根并由 $N$ 和 $M$ 决定, 模型数 $R=N$. 相比于 (10) 式, 该方法的使用更为便利, 当 $N$ 比较大 的时候 (12) 式的计算量大大减小, 但均匀度上无显著差异, 基于数论方法的均匀点集大多由该方法生 成, 所以 (12) 式可作为生成伪 Monte Carlo 模型集的主要方法之一.

根据上文所述, 伪 Monte Carlo 模型集的构造方法如下:

1. 根据模型的数量 $N=R$ 确定各参数的离散水平, 并根据 (9) 式获得各参数的离散水平;

2. 在 $N$ 为素数时根据 $N$ 与 $M$ 的值查表获得 $b$ 的值 ${ }^{[28]}$, 并根据 (12) 式设计模型集;

3. 当 $N+1$ 为素数时根据 $N+1$ 与 $M$ 的值获得 $b$ 的值, 根据 (12) 式生成模型集, 并将生成的模 型集中最后一个模型去掉获得 $N$ 个模型构成的模型集 ${ }^{[28]}$.

4. 其他情况下根据 $N$ 与 $M$ 的值获得向量 $\left(a_{1}, \ldots, a_{M}\right)$ 的值 ${ }^{[27]}$, 并根据 (10) 式生成模型集.

在以上方法生成的伪 Monte Carlo 模型集的基础上, 通过相应参数不同水平的合并可得到混合水 平的伪 Monte Carlo 模型集 ${ }^{[29]}$ : 首先, 若任意 $\theta_{i} \in \Theta$ 对应的参数水平数从 $N_{i}$ 减为 $N_{i}^{\prime}$, 则合并后的 水平数满足 $\bmod \left(R, N_{i}^{\prime}\right)=0$; 其次, 在合并得到的所有模型集矩阵中, 偏差最小的为混合水平伪 Monte Carlo 模型集.

\section{3 伪 Monte Carlo 模型集的属性}

伪 Monte Carlo 模型集的设计方法表明这种模型集实现了在模式空间的一维投影空间以及 $M$ 维 模式空间的均匀分布. 星偏差的定义及伪 Monte Carlo 模型集的生成方法表明了本文构造的伪 Monte Carlo 模型集是极小极大设计, 因而伪 Monte Carlo 模型集对模型不确定的系统具有鲁棒性.

根据上述引理 1 及王元所给的推论, 基于伪 Monte Carlo 模型集的估计器的精度取决于模型集的 势, 因而为了得到更小的误差可以增大模型集的势. 一般地, 可以使用等水平的伪 Monte Carlo 模型 集, 但是模型集势的增加必然导致估计器计算量的增加, 为了以较少的数量获得较小的估计误差, 需 要使模型集更符合系统的特征. 对于线性系统, 如果某些参数的取值范围大, 则需要增加该参数的离 散水平; 而对于非线性系统, 除了考虑参数的取值范围外, 还需要考虑各参数对系统的影响程度, 如果 模型集中包含的参数对系统输出有相同的影响程度, 则使用各个参数离散水平相等的伪 Monte Carlo 模型集; 如果各个参数对系统输出的影响程度不相等, 从数值积分的角度, 增加模型的势可以减少对 
系统非线性的依赖. 然而系统的实时性要求减少模型集的势, 因而采用包含较少模型数量的混合水平 伪 Monte Carlo 模型集.

\section{5 仿真实验}

本节给出数值仿真的结果. 再入的初始状态为高斯随机向量, 其均值和协方差为 $X_{0}=[232000$

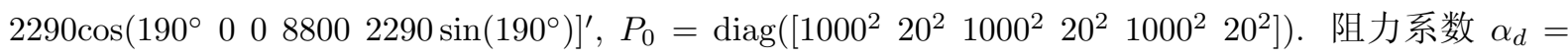
$\frac{9.8}{40000} \mathrm{~m}^{2} / \mathrm{kg}$, 升阻比与转阻比的区间为 $k_{c d} \in\left[\begin{array}{lll}-0.3 & 0.3\end{array}\right], k_{t d} \in\left[\begin{array}{ll}-0.3 & 0.3\end{array}\right]$. 雷达固定在地球表面上而且 到地心的距离等于地球的平均半径. 该雷达检测概率为 1 , 虚警概率为 0 , 采样间隔为 $1 \mathrm{~s}$, 量测量为径 向距离 $r$, 方位角 $b$, 和俯仰角 $e$,

$$
Z=\left[\begin{array}{lll}
r & b & e
\end{array}\right]^{\prime} .
$$

量测误差标准差为 $\sigma_{r}=100 \mathrm{~m}, \sigma_{b}=0.05 \mathrm{rad}, \sigma_{e}=0.05 \mathrm{rad}$.

仿真采用如表 1-3 所示的 3 个伪 Monte Carlo 模型集: $\mathrm{M}\left(6 ; 6^{3}\right), \mathrm{M}\left(12 ; 12^{3}\right), \mathrm{M}\left(18 ; 18^{3}\right)^{[29]}$. 因为 $6,12,18$ 所对应的 $N+1$ 都是素数, 所以先根据 $N+1$ 和 $M$ 的值查表获得 $b$ 的值分别为 3,4 , 和 8 , 根据 (12) 式生成模型集, 然后删除所生成模型集的最后一个模型即可得到表 1-3 所示的模型集. 另 外, 在模式空间中抽取势为 $6,12,18$ 的 Monte Carlo 模型集, 每次 Monte Carlo 仿真抽样生成这些模 型集, 然后这些模型集在跟踪过程中保持不变.

考虑到飞行器动力学模型的非线性和量测模型的非线性, 滤波器采用 Unscented filter (UF) ${ }^{[3,4,34]}$. UF 在状态的一步预测和预测更新时使用 unscented 变化, 其含义是采用一组满足特定条件 (比如满足 给定期望和方差) 的点 (sigma points), 这组点在理论上能捕捉到任何非线性函数后验均值和方差的二 阶项. 所有结果基于 100 次独立 Monte Carlo 仿真得到, 因为飞行器初始状态和各参数取值的不确定 性, 直到再入体触地才表示再入过程结束, 每次再入的时间长度为 107-158 s.

仿真比较了平均归一化均方差 (ANEES), 位置均方根 (RMS) 误差, 速度 RMS 误差和参数 RMS 误差 ${ }^{[15]}$ :

$$
A N E E S=\frac{1}{N n} \sum_{i=1}^{N}\left(X_{i}-\hat{X}_{i}\right)^{\prime} P_{i}^{-1}\left(X_{i}-\hat{X}_{i}\right),
$$

其中 $X_{i}-\hat{X}_{i}$ 和 $P_{i}$ 是第 $i$ 次 Monte Carlo 仿真的状态误差向量和协方差, $n$ 是状态向量的维数, $N$ 是 仿真的次数. 该指标的值越接近于 1 表示估计器的可信度越高.

模式中任一参数 $\theta$ 估计的 RMS 误差为 ${ }^{[15]}$

$$
\left\|\theta_{i}-\hat{\theta}_{i}\right\|^{2}=\frac{1}{N} \sum_{j=1}^{N}\left(\theta_{i j}-\hat{\theta}_{i j}\right)^{\prime}\left(\theta_{i j}-\hat{\theta}_{i j}\right),
$$

其中 $\theta_{j}$ 和 $\hat{\theta}_{j}$ 分别表示第 $j$ 次 Monte Carlo 仿真中 $\theta$ 的真值和估计值. 位置的 RMS 误差和速度的 RMS 误差由 (15) 式得到, 只需把参数替换为相应的位置向量或者速度向量即可.

从图 4 可以看出在再入的初始阶段各模型集估计的可信度相近, $70 \mathrm{~s}$ 之后伪 Monte Carlo 模型集 估计的可信度高于 Monte Carlo 模型集, 而模型集势的增加又导致相应类别模型集可信度的提高, 伪 Monte Carlo 模型集势为 6 和 12 时估计器的可信度接近于 Monte Carlo 模型集势为 12 和 18 时估计 器的可信度.

图 5 和 6 也反映出在再入的初始阶段 (70 s 之前) 不同模型集在基态估计的误差上没有明显的差 别; 而到 $70 \mathrm{~s}$ 之后这两种模型集估计误差变得明显, 伪 Monte Carlo 模型集构成的估计器的估计误差 
表 1 模型集 $\mathrm{M}\left(6 ; \mathbf{6}^{\mathbf{3}}\right)$

Table 1 Model Set M $\left(6 ; 6^{3}\right)$

\begin{tabular}{cccc}
\hline Model & Para. 1 & Para. 2 & Para. 3 \\
\hline 1 & 1 & 3 & 2 \\
2 & 2 & 6 & 4 \\
3 & 3 & 2 & 6 \\
4 & 4 & 5 & 3 \\
5 & 5 & 1 & 5 \\
6 & 6 & 4 & 3 \\
\hline
\end{tabular}

表 2 模型集 $\mathrm{M}\left(12 ; \mathbf{1 2}^{3}\right)$

Table 2 Model Set M $\left(12 ; 12^{3}\right)$

\begin{tabular}{cccc}
\hline Model & Para. 1 & Para. 2 & Para. 3 \\
\hline 1 & 1 & 4 & 3 \\
2 & 2 & 8 & 6 \\
3 & 3 & 12 & 9 \\
4 & 4 & 3 & 12 \\
5 & 5 & 7 & 2 \\
6 & 6 & 11 & 5 \\
7 & 7 & 2 & 8 \\
8 & 8 & 6 & 11 \\
9 & 9 & 10 & 1 \\
10 & 10 & 1 & 4 \\
12 & 11 & 5 & 7 \\
\hline
\end{tabular}

表 3 模型集 $\mathrm{M}\left(18 ; 18,6^{2}\right)$

Table 3 Model Set $\mathrm{M}\left(18 ; 18,6^{2}\right)$

\begin{tabular}{cccc}
\hline Model & Para. 1 & Para. 2 & Para. 3 \\
\hline 1 & 1 & 8 & 7 \\
2 & 2 & 16 & 14 \\
3 & 3 & 5 & 2 \\
4 & 4 & 13 & 9 \\
5 & 5 & 2 & 16 \\
6 & 6 & 10 & 4 \\
7 & 7 & 18 & 11 \\
8 & 8 & 7 & 18 \\
9 & 9 & 15 & 6 \\
10 & 10 & 4 & 13 \\
11 & 11 & 12 & 1 \\
12 & 12 & 1 & 8 \\
13 & 13 & 9 & 15 \\
14 & 14 & 17 & 3 \\
15 & 15 & 6 & 10 \\
17 & 16 & 14 & 5 \\
\end{tabular}




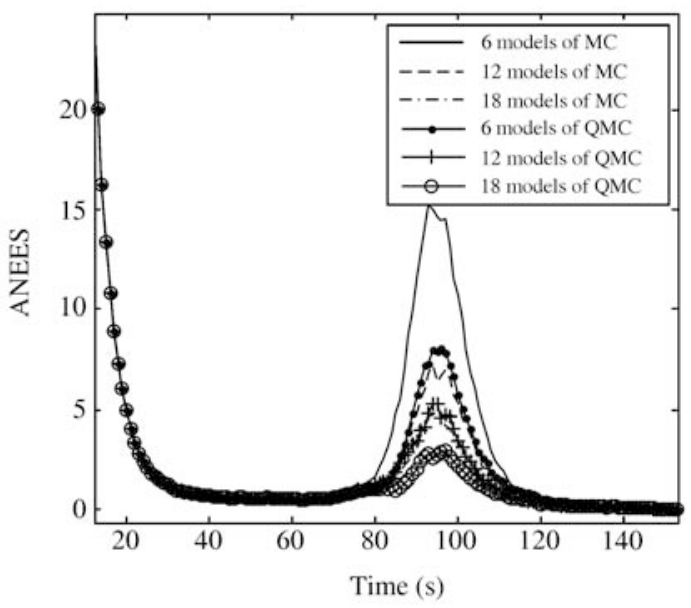

图 4 ANEES

Figure 4 ANEES

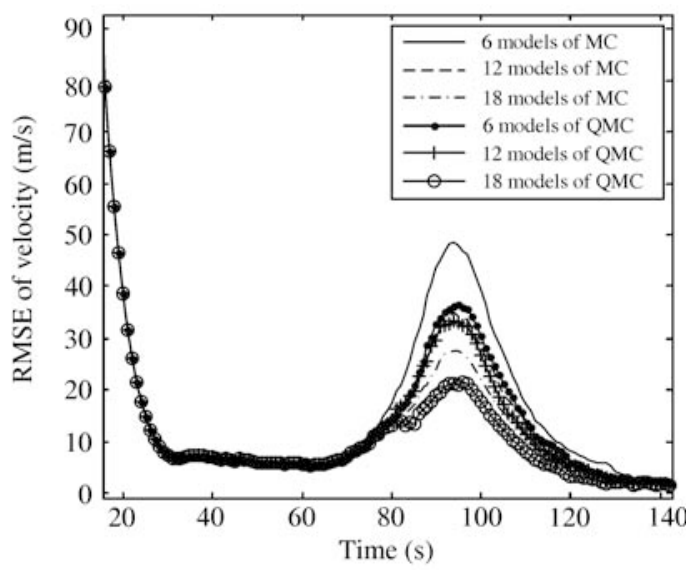

图 6 速度 RMS 误差估计

Figure 6 RMSE of velocity

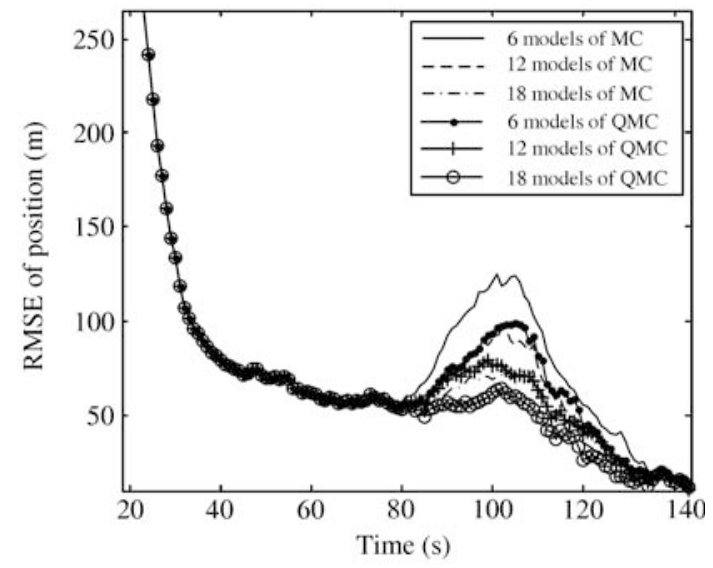

图 5 位置 RMS 误差估计

Figure 5 RMSE of position

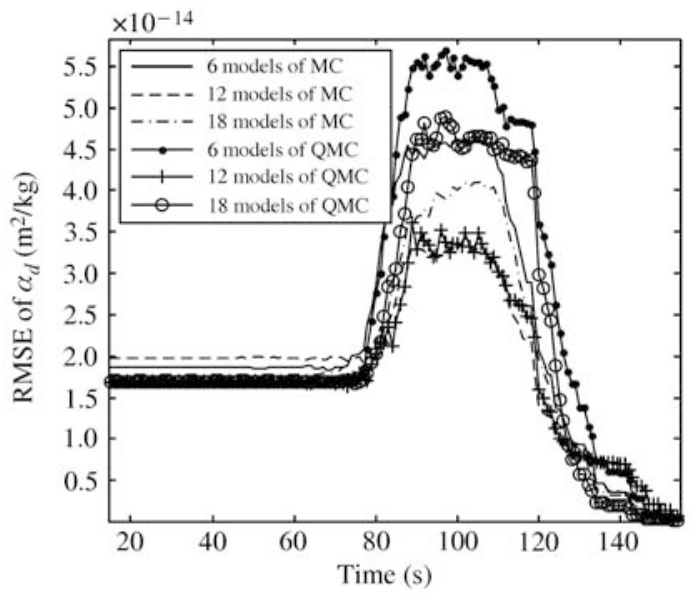

图 $7 \quad \alpha_{d} \mathrm{RMS}$ 误差估计

Figure 7 RMSE of $\alpha_{d}$

明显地低于相同势的 Monte Carlo 模型集, 前者势为 6 的估计误差相当于后者势为 12 的估计误差, 同 时前者势为 12 的估计误差相当于后者势为 18 的估计误差.

从 ANEES 和基态估计的结果也看出估计的可信度和精度受到模型集类别及其势的影响,伪 Monte Carlo 模型集比相同势的 Monte Carlo 模型集估计的可信度更高而误差更低, 对于给定的估计可信度 和估计误差, 伪 Monte Carlo 模型集相应于 Monte Carlo 模型集在势上大致节省三分之一.

从图 7-9 可看出在再入的初始阶段各模型集模式估计误差的差异小. 相比于 Monte Carlo 模型 集, 伪 Monte Carlo 模型集的估计误差更小, 而且其精度受模型集势的影响小很多. 在 $70 \mathrm{~s}$ 之后模式 估计的误差曲线波动剧烈, 伪 Monte Carlo 模型集的误差整体上低于 Monte Carlo 模型集. 从仿真结 果可以看出各参数估计的误差总体上随模型集势的增加而减小, 这是因为非线性系统的复杂性, 基态 估计与模式估计的误差与模型集势之间的关系难以做到完全一致.

上述仿真结果在再入的不同阶段差别很大, 这是因为再入过程中空气密度和飞行器速度的变化引 


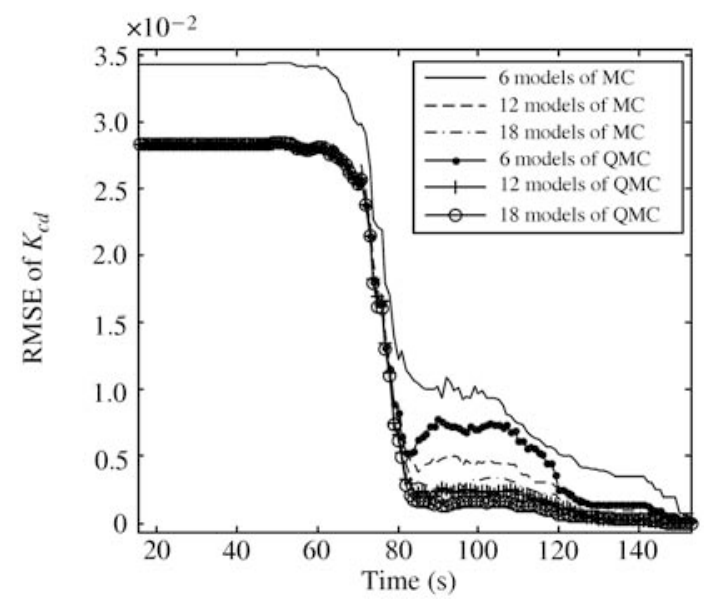

图 $8 k_{c d} \mathrm{RMS}$ 误差估计

Figure 8 RMSE of $k_{c d}$

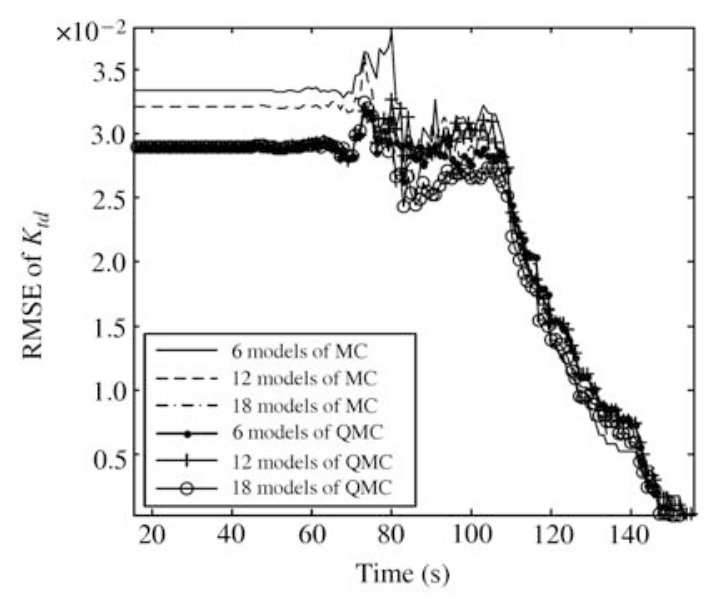

图 $9 k_{t d} \mathrm{RMS}$ 误差估计

Figure 9 RMSE of $k_{t d}$

起的. 在再入的初始阶段空气稀薄, 飞行器的状态受到气动力的影响很小, 随着再入的进行, 空气密度 逐渐增加, 而飞行器的速度依然很高, 因而气动力的作用越来越明显, 到一定时间之后 (对于本文的场 景来说是再入 $70 \mathrm{~s}$ 之后) 飞行器的状态因为气动力的作用而发生剧烈的变化, 因而其状态估计的精度 曲线会发生剧烈的波动, 各种模型集的差异也主要体现在这一阶段, 随着再入的继续进行, 飞行器的 速度被进一步降低, 因而其受到的气动力会继续减小, 状态估计的精度曲线变得相对平稳.

仿真结果符合理论分析的结论, 实际上, 因为再入模式的不确定性, 相比于 Monte Carlo 法生成的 模型集, 伪 Monte Carlo 模型集确保了每个模型在模式空间所代表的模式空间大致相等, 因而这种模 型集能更好地代表了未知模式, 从而使得该模型集对任意不确定的再入模式的估计结果不会太差.

虽然模型集势的增加可以减小估计的误差, 值得注意的是, 估计器实际需要的模型数量需要综合考 虑处理器的计算能力, 精度的要求和系统实时性的要求. 考虑到再入阻力系数的变化范围较大, 建议在 实际应用中可以增加阻力系数的离散水平, 在模型集上采用混合水平的模型集, 比如使用 $\mathrm{M}\left(12 ; 12,6^{2}\right)$, $\mathrm{M}\left(24 ; 24,12^{2}\right)$. 相比于从文献 [29] 直接获得的模型集, 为了获得更小的估计误差, 建议优先从文献 [28] 获得生成向量并根据 4.2 小节所述方法生成模型集. 另外, 基态估计器中存在的滤波环节而模式估计 器没有, 因而在状态变化剧烈情况下, 基态估计的鲁棒性好于模式估计, 同时基态估计精度和模型集 势之间的规律性好于模式估计.

\section{6 结论}

本文在分析不变结构 SBRV 运动特征和模式特征的基础上, 根据 MMSE 准则得到了该飞行器 的接近于最优的混合状态估计器, 在参数有界分布区间已知的前提下根据数论方法提出了伪 Monte Carlo 模型集, 并探讨了模型集的生成方法和性能特征. 这种模型集即适用于参数张成的多维模式空 间, 又适用于非线性的不变结构 SBRV 的再入估计. 理论及仿真结果表明这种模型集相比于相同势的 Monte Carlo 模型集具有更高的可信度和更小的估计误差. 


\section{参考文献}

1 Li X R, Jilkov V P. A survey of maneuvering target tracking-Part II: Ballistic target models. In: Proc of 2001 SPIE Conf on Signal and Data Processing of Small Targets, San Diego, 2001. 559-581

2 Mehra R K. A comparison of several nonlinear filters for reentry vehicle tracking. IEEE Trans Automat Contr, 1971, 16: $307-319$

3 Farina A, Ristic B, Benvenuti D. Tracking a ballistic target: Comparison of several nonlinear filters. IEEE Trans Aerospace Electron Syst, 2002, 38: 854-867

4 Zhao Z L, Chen H M, Chen G S, et al. IMM-LMMSE filtering algorithm for ballistic target tracking with unknown ballistic coefficient. In: Proc 2006 SPIE Conf on Signal and Data Processing of Small Targets, Orlando, 2006. 62360K$1-62360 \mathrm{~K}-11$

5 Wang X J. Reentry Technology for Spacecraft (in Chinese). Beijing: Chinese Aerospace Press, 2006. 1-151

6 Burkhardt J, Schottle U. Flight performance and control aspects of a semi-ballistic reentry capsule. In: Proc of AIAA Atmospheric Flight Mechanics Conference, San Diego, 1996. 1-11

7 Zhou F Q, Cui L M, Zhou J. The control of ballistic arhead with variable centroid (in Chinese). Astronautics J, 2000, 21: $107-110$

8 Yi Y, Zhou F Q. Variable centroid control scheme over hypersonic tactical missile. Sci China Ser G-Phys Mech Astron, 2003, 46: 561-569

9 Liang Y Q, Han C Z, Yang Y A. Modeling and estimation to semi-ballistic reentry vehicle. In: Proc of the International Colloquium on Information Fusion, Xi'an, 2007. 122-130

10 Liang Y Q, Han C Z. Tracking of semi-ballistic reentry vehicle. In: Proc of IEEE International Symposium on Intelligent Control, San Antonio, 2008. 389-394

11 Li X R, Jilkov V P. Survey of manuvering target tracking. Part V: Multiple-model method. IEEE Trans Aerosp Electron Syst, 2005, 41: 1255-1321

12 Han C Z, Zhu H Y, Duan Z S. Multi-source Information Fusion (in Chinese). Beijing: Tsinghua University Press, 2006. $16-67$

13 Li X R. Optimal selection of estimate for multiple-model approach with uncertain parameters. IEEE Trans Aerosp Electron Syst, 1998, 34: 653-657

14 Magill D T. Optimal adaptive estimation of sampled stochastic processes. IEEE Trans Automat Contr, 1965, 10: 434-439

15 Bar-Shalom Y, Li X R, Kirubarajan T. Estimation with Application to Tracking and Navigation. New York: Wiley, 2001. 199-266

16 Baram Y, Samdel N R. An information theoretic approach to dynamical systems modeling and identification. IEEE Trans Automat Contr, 1978, 23: 61-66

17 Li X R, Zhao Z L, Li X B. General model-set design method for multiple-model approach. IEEE Trans Automat Contr, 2005, 50: 1260-1276

18 Sheldon S N, Maybeck P S. An optimizing design strategy for multiple model adaptive estimation and control. IEEE Trans Automat Contr, 1993, 38: 651-654

19 Liang Y Q, Li X R, Han C Z, et al. Model-set design: Uniformly distributed models. In: Proc of the 48th IEEE Conference on Decision and Control, Shanghai, 2009. 39-44

20 Sobol I M. An exact estimate of the error in multidimensional quadrature formulas for functions of the classes W1 and H1. Z Zycisl Mat I Mat Fiz, 1961, 1: 208-216

21 Bahvalov N S. On approximate computation of multiple integrals. Vestnik Moskov Univ Ser Mat Mekh Astr Fiz Khim, 1959, 1: $3-18$

22 Roth K F. On irregularities distribution. Mathematica, 1954, 2: 73-79

23 Wang Y, Yang C C, Pan C B. Number theoretic method in numerical integration. Contemp Math AMS, 1988, 77: 63-81

24 Hua L K, Wang Y. On uniform distribution and numerical analysis I (number-theoretic analysis). Sci China Ser A-Math, 1973, 16: 483-505 
25 Hua L K, Wang Y. On uniform distribution and numerical analysis II (number-theoretic analysis). Sci China Ser A-Math, 1974, 17: 331-348

26 Hua L K, Wang Y. On uniform distribution and numerical analysis III (number-theoretic analysis). Sci China Ser AMath, 1975, 18: 184-198

27 Hua L K, Wang Y. Application of Number Theory to Numerical Analysis (in Chinese). Beijing: Science Press, 1978. $48-114$

28 Wang Y, Fang K T. A note on uniform distribution and experimental design (in Chinese). Chin Sci Bull, 1981, 26: 486-489

29 Fang K T. Uniform Design and Uniform Tables (in Chinese). Beijing: Science Press, 1994. 35-52

30 Julier S, Uhlmann J, Durrant-Whyte H. A new method for nonlinear transformation of means and covariances in filters and estimators. IEEE Trans Automat Contr, 2000, 45: 477-482

31 Korobov N M. The approximate computation of multiple integrals. Dokl Akad Nauk SSSR, 1959, 124: 1207-1210

32 Hlawka E. Zur angenaherten Berechnumg mehrfacher integrale. Monatsh Math, 1962, 66: 140-151

33 Korobov N M. Computation of multiple integrals by the method of optimal coefficients. Vestnik Moskow Univ Ser Mat Astr Fiz Him, 1959, 4: 19-25

34 Niederreiter H. Pseudorandom numbers and optimal coefficients. Adv Math, 1977, 26: 99-181

\title{
Hybrid state estimation and model-set design of invariable- structure semi-ballistic reentry vehicle
}

\author{
LIANG YongQi ${ }^{1}$, HAN ChongZhao ${ }^{2}$, SUN YaoJie ${ }^{3 *} \&$ LIN YanDan ${ }^{3 *}$ \\ 1 School of Aerospace, Xi'an Jiaotong University, Xi'an 710049, China; \\ 2 MOE KLINNS Lab, SKLMSE Lab, Institute of Integrated Automation, School of Electronics and Information \\ Engineering, Xi'an Jiaotong University, Xi'an 710049, China; \\ 3 Department of Light Sources and Illuminating Engineering, Engineering Research Center of Advanced Lighting \\ Technology, Ministry of Education, Fudan University, Shanghai 200433, China \\ *E-mail: yjsun@fudan.edu.cn, ydlin@fudan.edu.cn
}

\begin{abstract}
Multiple-model approach is one of the main streams for hybrid estimation. The difficulty of this approach to estimate the hybrid state of the semi-ballistic reentry vehicle (SBRV) is model-set design. This paper proposes a quasi-Monte Carlo model set that can ensure the estimator near-optimal in the sense of minimum mean square error (MMSE). The SBRV has a high nonlinearity and its mode is spanned by multiple parameters with known bounds. The design methods and characteristics of the quasi-Monte Carlo model set are given. The proposed model set has a higher accuracy than the model-set generated by the Monte Carlo method. The theoretical analysis and simulation results show the effectiveness and reasonability of the newly designed model set.
\end{abstract}

Keywords semi-ballistic reentry vehicle, hybrid estimation, model-set design, Monte Carlo method, quasiMonte Carlo method 